\title{
Effects of peri-operative intravenous administration of dexmedetomidine on emergence agitation after general anesthesia in adults: a meta-analysis of randomized controlled trials
}

This article was published in the following Dove Press journal:

Drug Design, Development and Therapy

\author{
Jian Zhang ${ }^{1, *}$ \\ Yang $\mathrm{Yu}^{\mathrm{l}}, *$ \\ Shuai Miao ${ }^{2}$ \\ Lu Liu' \\ Shuyuan Gan' \\ Xianhui Kang' \\ Shengmei Zhu' \\ 'Department of Anesthesiology, The \\ First Affiliated Hospital, College of \\ Medicine, Zhejiang University, Hangzhou, \\ Zhejiang, People's Republic of China; \\ ${ }^{2}$ Department of Anesthesiology, The \\ Affiliated Hospital, Xuzhou Medical \\ University, Xuzhou, China \\ *These authors contributed equally to \\ this work
}

Correspondence: Xianhui Kang; Shengmei Zhu

Department of Anesthesiology, The First Affiliated Hospital, College of Medicine,

Zhejiang University, No. 79 Qingchun

Road, Hangzhou, Zhejiang, People's

Republic of China

Tel +86057187236169

Fax +862157643271

Email kxhui66@zju.edu.cn;

smzhu20088@zju.edu.cn
Objective: The aim of the current meta-analysis was to assess the effect of dexmedetomidine on emergence agitation (EA) and the recovery outcomes after general anesthesia in adults.

Methods: We searched the PubMed, the Cochrane Central Register of Controlled Trials, Embase, Web of Science and clinicaltrials.gov for relevant randomized controlled trials (RCTs) investigating the effects of dexmedetomidine on the EA in adults after general anesthesia compared with placebo. The primary outcome was the incidence of EA. Secondary outcomes included other recovery outcomes after general anesthesia.

Results: Twelve RCTs (842 participants) met the eligibility criteria. A conventional randomeffects meta-analysis demonstrated that peri-operative intravenous dexmedetomidine could be effective for the prevention of EA [risk ratio (RR) 0.49, Trial Sequential Analysis (TSA)adjusted $95 \%$ confidence interval (CI) $0.35-0.68, P<0.00001]$. In addition, the TSA indicated that the meta-analysis for the incidence of EA reached the required information size (370). Lower number of patients receiving dexmedetomidine required analgesia $(P=0.0009)$. Extubation time was longer $(P=0.03)$ and hypotension $(P=0.03)$ was more common with dexmedetomidine. Moreover, no difference was found in the other outcomes.

Conclusion: Dexmedetomidine was shown to effectively decrease the incidence of EA and to reduce postoperative analgesic requirements. Yet, other recovery outcomes including extubation time, length of PACU stay, postoperative residual sedation, hypotension, bradycardia as well as postoperative nausea and vomiting provided no data that could be used to form final conclusions.

Keywords: dexmedetomidine, emergence agitation, adults, general anesthesia, metaanalysis

\section{Introduction}

Emergence agitation (EA), also known as emergence delirium, is defined as a transient impairment of consciousness that occurs during the early stage after general anesthesia. ${ }^{1,2}$ It is now considered as a common postoperative complication found in children and adults in post-anesthesia care unit (PACU). ${ }^{3,4}$ Previous studies have suggested that the prevalence of EA in adults varies from $4.7 \%$ to $21.3 \%{ }^{3,5,6}$ EA has complex manifestations that can suddenly become dangerous and lead to serious consequences, such as increased pain intensity, hemorrhage, accidental self-extubation 
and catheter removal. ${ }^{5}$ In addition, even EA in the recovery room could help to predict postoperative delirium after hipfracture repair and has been associated with increased total length of hospital stay. ${ }^{7,8}$ Thus, strategies have been sought to reduce the incidence of EA and improve the postoperative conditions with various measurements. ${ }^{9,10}$

As a highly specific $\alpha_{2}$-adrenoreptor agonist, ${ }^{11,12} \mathrm{dex}$ medetomidine is considered an effective drug to treat EA in children. ${ }^{13-15}$ However, there are few systematic reviews and meta-analysis on the use of dexmedetomidine for prevention of EA in adults. ${ }^{16}$ Recently, quite a few studies have examined the effect of different infusion modes of dexmedetomidine on EA, which were unfortunately marked by substantial clinical and statistical heterogeneities that might undermine a precise estimation of the total dexmedetomidine treatment effect. ${ }^{10,17,18}$

Consequently, this meta-analysis was conducted to assess the efficacy and safety of dexmedetomidine for preventing EA in adults after general anesthesia.

\section{Materials and methods}

\section{Protocol and registration}

The current meta-analysis was conducted and presented in accordance with the criteria of the PRISMA statement and the current recommendations of the Cochrane Collaboration. ${ }^{19-21}$ The protocol was registered in the PROSPERO (ID: CRD42017065915).

\section{Inclusion and exclusion criteria}

Articles that met the following criteria were included: 1) original and independent randomized controlled trial (RCT), 2) the interventions that included treatment with dexmedetomidine versus placebo (normal saline) regardless of administration modes; and 3) patients $>18$ years old with American Society of Anesthesiologists (ASA) physical status of I-III. Exclusion criteria: 1) patients with cognitive dysfunction; 2) patients undergoing neurosurgery or cardiac surgery; 3) dexmedetomidine administrated via any non-intravenous route, such as intravertebral or intranasal routes; and 4) abstracts or articles without complete data.

\section{Search strategy and study selection}

Two authors independently searched and retrieved relevant studies from the following databases: PubMed, EMBASE, Web of Science and Cochrane Library. This search strategy combined medical subject headings (MeSH), Emtree text words and controlled vocabulary terms relating to dexmedetomidine and EA that were limited to Englishlanguage literature for practical reasons. Details of the search strategy are presented in the Appendix. The most recent search was performed on October 25, 2017. In addition to the planned literature search, we have also examined www.clinicaltrials.gov for completed studies.

The titles and article abstracts were independently identified, and any disagreement between the authors was settled by discussion with a third author until a consensus was reached. If there was a potential correlation, a full-text search was performed. During the review, the reasons for the exclusion of each publication were indicated. In addition, reference lists of relevant reviews, meta-analysis and included studies were examined to identify potentially relevant additional studies. Endnote X7 was used for combining and removing duplicate citations.

\section{Data extraction and outcome measures}

The data extraction was conducted independently by two co-authors. Any discrepancies were resolved by reexamining the source data as a first resort, followed by consulting with a third author. The following study characteristics and relevant clinical outcomes were extracted: the first author, year of publication, sample size, age, type of surgery, ASA status, detailed intervention of dexmedetomidine and outcomes of interest.

\section{A priori hypothesis for sources of variability in effect sizes}

We made a priori hypothesis for sources of variability in effect sizes and we considered the following factors that may affect the efficacy of dexmedetomidine on EA: 1) age of participants, 2) type of anesthesia, 3) timing of the administration of dexmedetomidine, 4) type of surgery and 5) sample size. Therefore, we tested whether these factors affected the efficacy of dexmedetomidine and if they could explain the heterogeneity in the study.

\section{Risk of bias in individual studies}

The quality of the included trials was independently assessed by two authors according to the guidelines of the Cochrane Reviewer's Handbook. ${ }^{22}$ The following six aspects were assessed: 1) sequence generation, 2) concealment of allocation, 3) method of blinding, 4) completeness of outcome data, 5) selective outcome reporting and 6) other sources of bias. 


\section{Data analysis}

Summary measures and synthesis of results

Random-effects models were used to determine the dexmedetomidine effect a priori for random-effects models dealing more rationally with heterogeneity. ${ }^{23}$ For dichotomous outcomes, the relative effect sizes were calculated as relative risk (RR) with $95 \%$ confidence intervals $(95 \%$ CIs). For continuous outcomes, standard mean difference (SMD) with $95 \%$ CIs was performed. The heterogeneity $\chi^{2}$ was calculated as the $\mathrm{I}^{2}$ for the variation due to heterogeneity. ${ }^{24,25}$

Due to sparse data and the repeated testing of cumulative data, and since meta-analysis may bring about type I errors, trial sequence analysis (TSA) was applied for primary outcomes. Trial sequential analysis was performed to estimate the necessary sample size for the meta-analysis to be reliable and conclusive, and to determine the statistical boundaries for efficacy and futility. ${ }^{26-30}$ The required information size (RIS) and the trial sequential monitoring boundaries (the statistical significance test before reaching the RIS) provided relevant information necessary to estimate the level of evidence for an experimental intervention and whether additional evidence and further trials were needed. Two-sided tests with type I error of 5\% and power of $90 \%$ were used. We performed random-effects models for analysis, and the adjusted $95 \%$ CI was calculated at the same time. Computational problems occurred if no events were observed in one or both groups in an individual study. We used a constant continuity correction of 0.01 to process "zero event" tests by increasing the continuity correction factor for the number of events and non-events in each intervention group by using the TSA software v.0.9.5.10 Beta. We made a priori hypothesis that dexmedetomidine might significantly reduce the incidence of EA and we calculated the relative risk reduction (RRR) and the incidence of control group (Pc) based on the raw data from the meta-analysis for the outcome $\mathrm{EA}(\mathrm{RRR}=52.6 \%, \mathrm{Pc}=45.9 \%)$.

\section{Publication bias}

Funnel plots were proposed as means for detecting publication bias, while the exaggeration of treatment effects in small studies of low quality provided a plausible alternative mechanism for funnel-plot asymmetry. ${ }^{31}$ Small-study effects can be observed due to real differences in the relative effectiveness in small and large trials. Therefore, the funnel plots were used for the qualitative data and
Egger's regression test for quantitative data and smallstudy effects. ${ }^{32}$ To disentangle the effects of heterogeneity caused by sample size and publication bias, we used a contour-enhanced funnel plot. ${ }^{33}$

\section{Meta-regression, subgroup analysis and sensitivity analysis}

Regression analysis was performed according to the a priori hypothesis to explore whether the aforementioned factors affected the effectiveness of dexmedetomidine and whether these variables were partially involved in the observed heterogeneity. The results from meta-regression are based on observational evidence, and since these studies cannot be randomized for specific characteristics, the results should be interpreted with caution. We aimed to explain the heterogeneity of the studies through these covariates. ${ }^{34}$ Based on the results of meta-regression analysis, we performed subgroup analysis to quantitatively explore the effectiveness of drug across different subgroups.

A recent study has indicated that the estimation of the outcome in the meta-analysis differs depending on the method used, which may lead to significant changes in the outcome. ${ }^{35}$ Sensitivity analysis was used to overcome this problem, by repeating the analysis after removing one study at a time, which showed the effect of each study on the overall effect size and heterogeneity.

\section{Software used}

RevMan 5.3 (The Cochrane Collaboration, Copenhagen, 2014) was used to carry out traditional meta-analysis, assess the risk of bias and draft a PRISMA flow diagram. The metaregression and publication bias were analyzed in STATA version 12.0 (STATA Corp., USA). The TSA software version 0.9.5.10 beta (Copenhagen Trial Unit, Denmark, 2011) was used for TSAs.

\section{Results}

After a systematic retrieval, 694 relevant documents were identified. Only 30 RCTs were included as potentially eligible after reviewing of the titles and abstracts. After full-text search and review, 10 clinical studies were consistent with our inclusion criteria., ${ }^{2,17,18,36-42}$ After the citation search, another RCT was identified. ${ }^{43}$ Therefore, 11 studies including 12 RCTs (842 participants) resulted eligible and were included in the study. The PRISMA flow diagram is shown in Figure 1. 


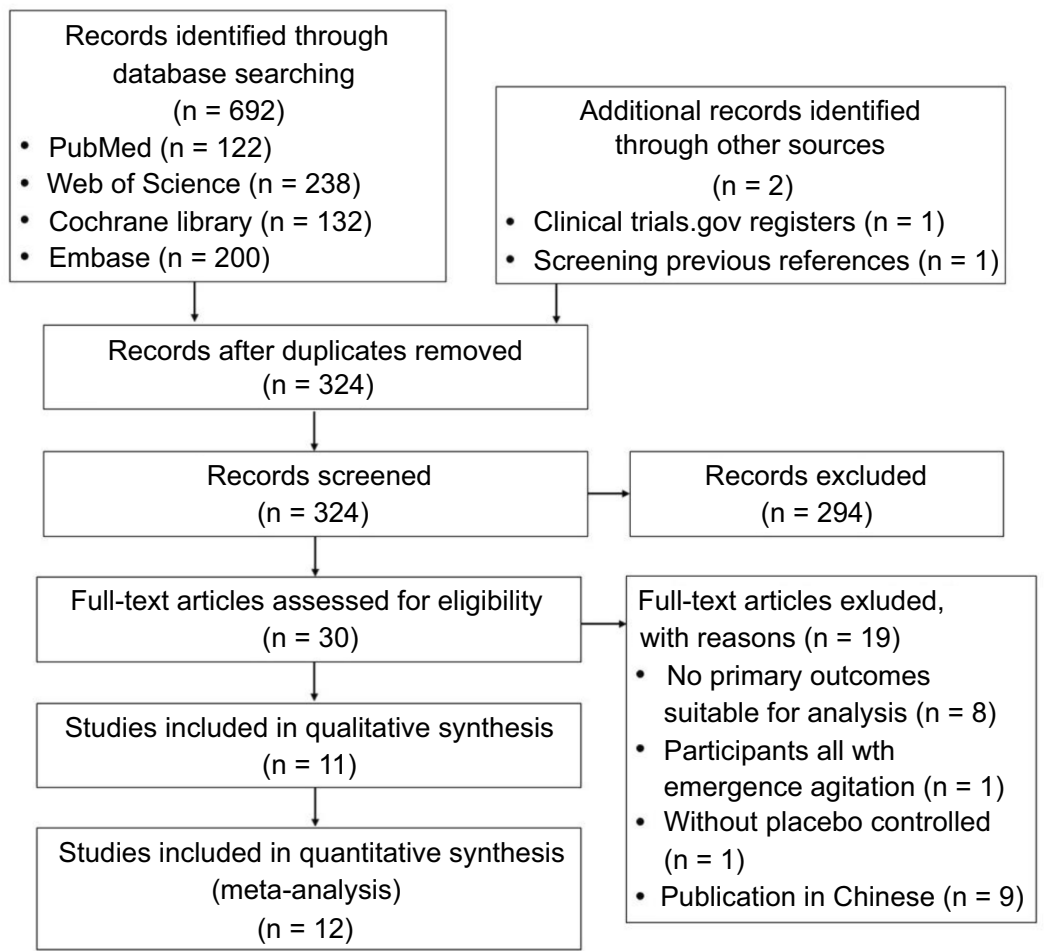

Figure I Flow diagram of the literature search.

\section{Characteristics of the studies included}

The detailed characteristics of the included studies are presented in Table 1. Several types of surgery were included: ENT surgery, ${ }^{36-38,42,43}$ oral and maxillofacial surgery, ${ }^{18}$ orthopedic surgery, ${ }^{2,17}$ thoracic surgery, ${ }^{40}$ gastrointestinal surgery ${ }^{41}$ and urological surgery. ${ }^{39}$ Patients were kept in the PACU after surgery in all trails. The paper of Kim et al was divided into two RCTs in accordance with methods of anesthesia; ${ }^{2}$ where one was performed with total intravenous anesthesia, and the other with intravenous-inhalation combined anesthesia.

\section{Risk of bias in included studies}

Using the guidelines from the Cochrane Reviewer's Handbook, our team assessed the risk of bias for each RCT included in the study. Cochrane risk of bias analysis is shown in Figure 2.

\section{Publication bias}

A visual inspection of the funnel plot showed an asymmetry. This finding was further evaluated by conducting Eggers test for small-study effects (regression line showing the association between SE and effect size embedded in the funnel plot), which gave a $P$-value of 0.001 , clearly suggesting the presence of small study effects (Figure 3A). Small-study effects may be caused by publication bias or true differences between small and large studies. By drawing a contour-enhanced funnel plot, small nonpositive studies were missing (from the white area), thus suggesting that the omission of small negative studies may be causing asymmetry in the funnel plot (Figure 3B).

\section{Meta-analysis of outcomes} Incidence of emergence agitation

Twelve RCTs (842 participants) reported the incidence of EA. The results suggested that dexmedetomidine could decrease the incidence of EA in patients after general anesthesia (RR $0.49,95 \%$ CI $0.36-0.66, P<0.00001, \mathrm{I}^{2}=49 \%$ ). Sensitivity analysis did not find any source of heterogeneity among studies.

Based on the a priori hypothesis and principles of meta-regression, we conducted a meta-regression analysis of the relevant studies from three perspectives: types of anesthesia (total intravenous anesthesia TIVA ${ }^{2,41}$ or intravenous-inhalation combined anesthesia $^{2,17,18,36-40,42,43}$ ), timing of administration of dexmedetomidine (pre-operative ${ }^{2,36,38,39,41,43 /}$ intraoperative $^{37,42 /}$ postoperative $\mathrm{e}^{17,18,40}$ ) and sample size $\left(\leq 60^{2,37-39,41,42}\right.$ or $\left.>60^{17,18,36,40,43}\right)$. The analysis showed 


\begin{tabular}{|c|c|c|c|c|c|c|c|c|c|c|c|c|c|c|c|c|c|c|c|}
\hline \multicolumn{2}{|c|}{ 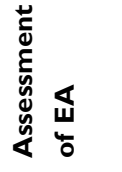 } & \multicolumn{2}{|l|}{ 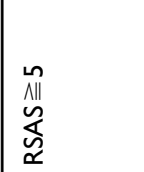 } & \multicolumn{2}{|l|}{ 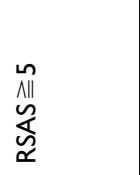 } & \multicolumn{2}{|l|}{ 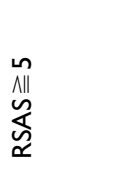 } & \multicolumn{2}{|l|}{ 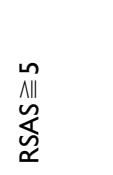 } & \multicolumn{2}{|c|}{ 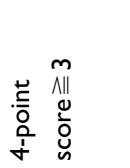 } & \multicolumn{2}{|l|}{$\begin{array}{l}\text { n } \\
\Lambda \| \\
\hat{\omega} \\
\alpha \\
\alpha\end{array}$} & \multicolumn{2}{|l|}{ 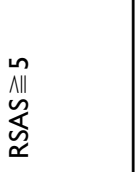 } & \multicolumn{2}{|l|}{ 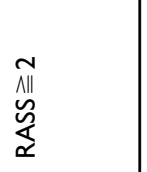 } & \multicolumn{2}{|l|}{ 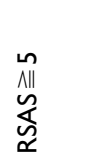 } \\
\hline \multicolumn{2}{|c|}{ 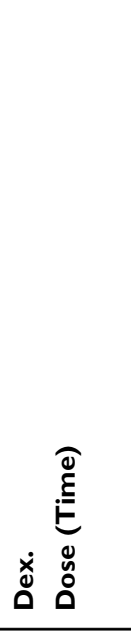 } & \multicolumn{2}{|c|}{ 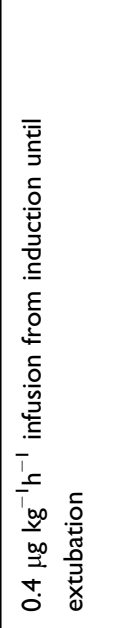 } & \multicolumn{2}{|c|}{ 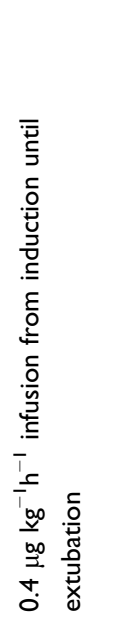 } & \multicolumn{2}{|c|}{ 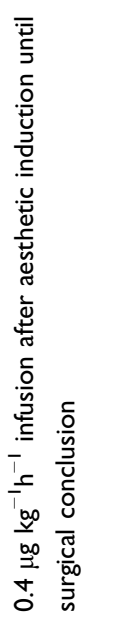 } & \multicolumn{2}{|c|}{ 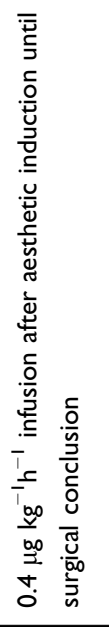 } & \multicolumn{2}{|c|}{ 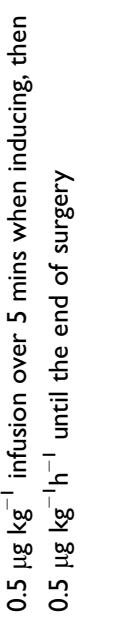 } & \multicolumn{2}{|c|}{ 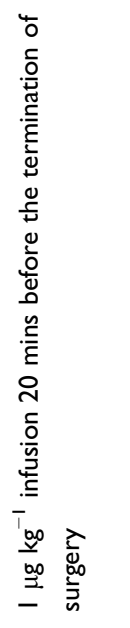 } & \multicolumn{2}{|l|}{ 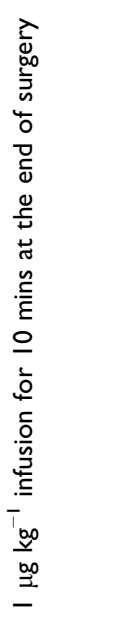 } & \multicolumn{2}{|l|}{ 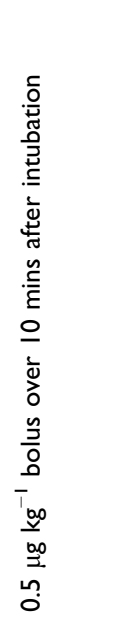 } & \multicolumn{2}{|c|}{ 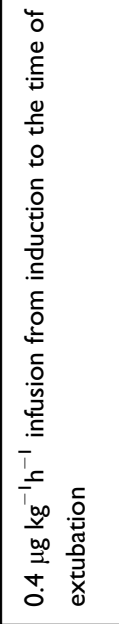 } \\
\hline \multicolumn{2}{|c|}{ 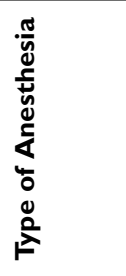 } & \multicolumn{2}{|c|}{ 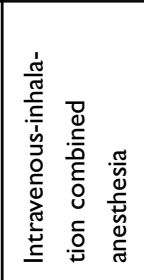 } & \multicolumn{2}{|c|}{ 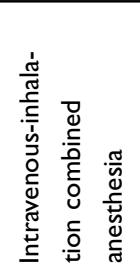 } & \multicolumn{2}{|c|}{ 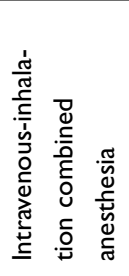 } & \multicolumn{2}{|c|}{ 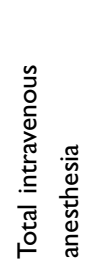 } & \multicolumn{2}{|c|}{ 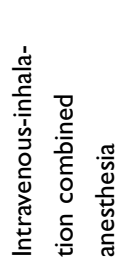 } & \multicolumn{2}{|c|}{ 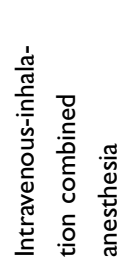 } & \multicolumn{2}{|c|}{ 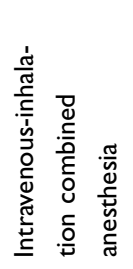 } & \multicolumn{2}{|c|}{ 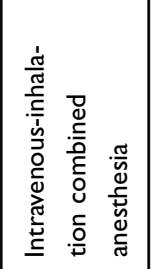 } & \multicolumn{2}{|c|}{ 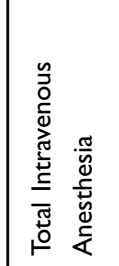 } \\
\hline \multicolumn{2}{|c|}{ 育 } & \multicolumn{2}{|l|}{$\overline{\bar{I}}$} & \multicolumn{2}{|l|}{$\overline{\bar{I}}$} & \multicolumn{2}{|l|}{$\overline{\bar{I}}$} & $\overline{\bar{I}}$ & & $\equiv$ & & $\stackrel{\equiv}{\equiv}$ & & $\overline{\bar{I}}$ & & $\overline{\bar{I}}$ & & $\overline{\bar{I}}$ & \\
\hline 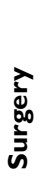 & & 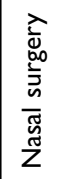 & & 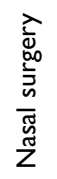 & & 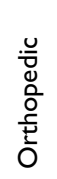 & & 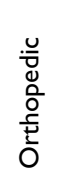 & & 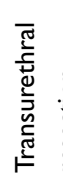 & & 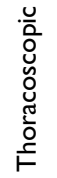 & & 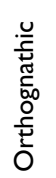 & & 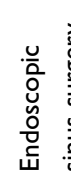 & 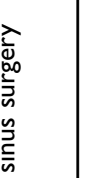 & 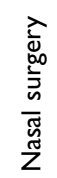 & \\
\hline & نَ & ᄒ্ল & & ᄒ̀n & & ন & & ᄒे & & ᄒे & & ᄒ̀ & & 焉 & & $\overline{\mathrm{m}}$ & & 훙 & \\
\hline $\mathfrak{\S}$ & ช̊ & & 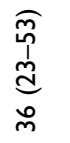 & & $\begin{array}{l}\text { } \\
\stackrel{0}{0} \\
\stackrel{d}{d} \\
m \\
m\end{array}$ & & $\begin{array}{l}\text { J } \\
\text { +1 } \\
\stackrel{N}{N} \\
\end{array}$ & & 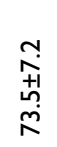 & & $\begin{array}{l}\hat{\sigma} \\
\frac{1}{+1} \\
\text { in }\end{array}$ & & 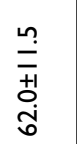 & & 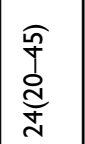 & & 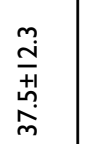 & & $\begin{array}{l}\text { Oे } \\
\text { dे }\end{array}$ \\
\hline 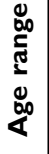 & $\stackrel{x}{\stackrel{x}{~}}$ & $\begin{array}{l}\stackrel{\tilde{L}}{0} \\
\underline{\infty}\end{array}$ & 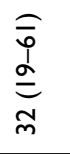 & $\begin{array}{l}\stackrel{\infty}{\hat{~}} \\
\stackrel{\text { d }}{2}\end{array}$ & $\begin{array}{l}\text { } \\
\stackrel{0}{0} \\
\stackrel{0}{d} \\
\text { c్ }\end{array}$ & $\stackrel{\sim}{0}$ & 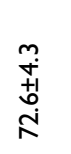 & 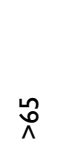 & 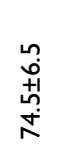 & $\begin{array}{l}\infty \\
\infty \\
0 \\
0\end{array}$ & $\begin{array}{l}1 \\
\infty \\
+ \\
+ \\
0 \\
0\end{array}$ & ્ㅗ & 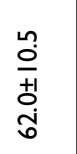 & 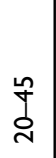 & 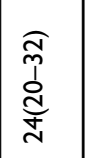 & $\begin{array}{l}0 \\
\stackrel{1}{0} \\
\grave{1}\end{array}$ & $\begin{array}{l}\stackrel{n}{\bar{n}} \\
\stackrel{+}{+1} \\
\stackrel{a}{+}\end{array}$ & $\begin{array}{l}\text { O } \\
\stackrel{1}{0} \\
\stackrel{1}{2}\end{array}$ & $\begin{array}{l}\text { o } \\
\stackrel{1}{\text { N }}\end{array}$ \\
\hline نे & & 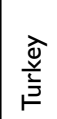 & & $\begin{array}{l}\widetilde{\Xi} \\
\stackrel{\Xi}{0} \\
\underline{y}\end{array}$ & & $\begin{array}{l}\stackrel{\Xi}{0 ّ} \\
\stackrel{0}{0}\end{array}$ & & $\begin{array}{l}\underset{\tilde{J}}{0} \\
\stackrel{0}{0}\end{array}$ & & $\begin{array}{l}\stackrel{\Xi}{0} \\
\stackrel{0}{0}\end{array}$ & & $\begin{array}{l}\widetilde{\Xi} \\
\stackrel{\tilde{\nu}}{0}\end{array}$ & & 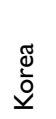 & & $\stackrel{\stackrel{\tilde{E}}{\bar{U}}}{\text { U. }}$ & & 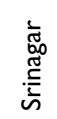 & \\
\hline 忞 & & $\begin{array}{l}\frac{\pi}{2} \\
\frac{0}{0} \\
\infty \\
\frac{0}{\pi} \\
\tilde{0} \\
\frac{0}{0} \\
\frac{\pi}{0}\end{array}$ & & 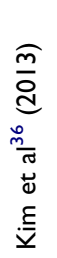 & & 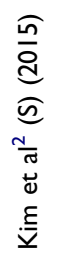 & & 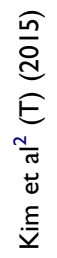 & & 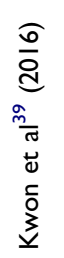 & & 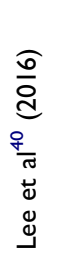 & & 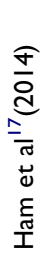 & & $\begin{array}{l}\widehat{0} \\
\frac{0}{0} \\
\frac{d}{\pi} \\
\frac{\pi}{0} \\
\dot{x}\end{array}$ & & $\begin{array}{l}\frac{\pi}{2} \\
\frac{d}{2} \\
\frac{m}{\pi} \\
\frac{0}{0} \\
\stackrel{0}{\frac{\pi}{1}}\end{array}$ & \\
\hline
\end{tabular}




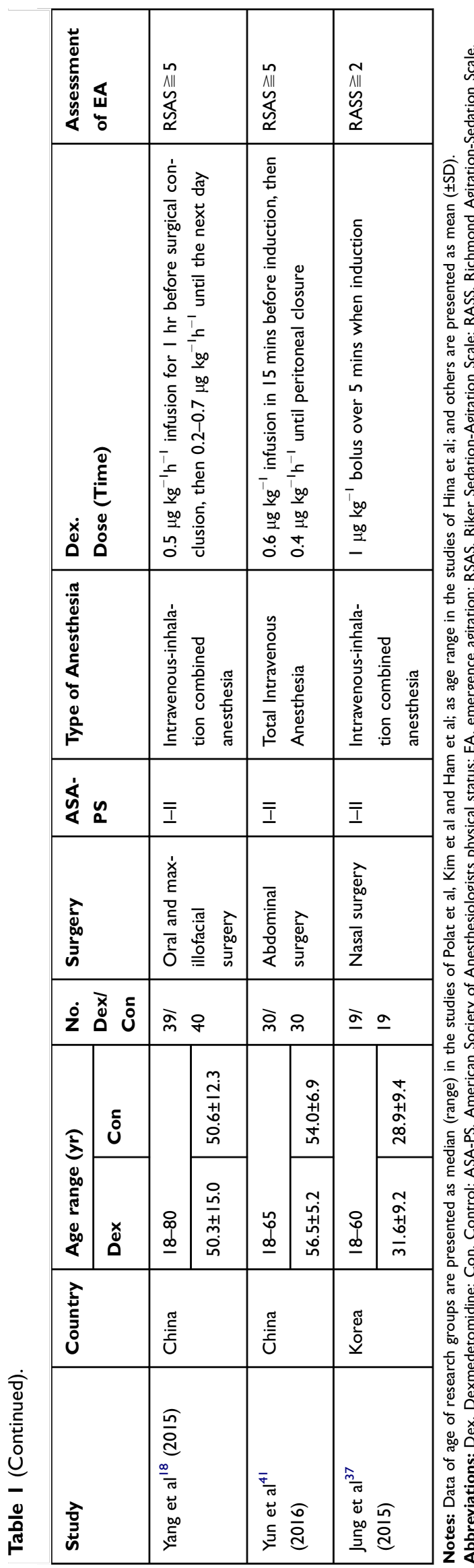

that different timing usage of dexmedetomidine was the main factor affecting heterogeneity $(P=0.026$; Table S1). Therefore, we performed a subgroup analysis. Interestingly, postoperative intravenous dexmedetomidine did not significantly reduce the occurrence of agitation in PACU (RR $0.75,95 \%$ CI $0.42-1.35, P=0.35$, $\mathrm{I}^{2}=43 \%$; Figure 4 ), while pre-operative or intra-operative intravenous dexmedetomidine could reduce the occurrence of agitation and reach statistical significance.

Considering age as an impact factor for EA, a subgroup analysis was conducted to evaluate the effect of different age levels $\left(\geq 65 \mathrm{yr}^{2}\right.$ or $\left.<65 \mathrm{yr}^{17,36-38,41-43}\right)$. Yet, no significant differences existed between the subgroups, while heterogeneity was large $\left(\mathrm{I}^{2}=87.1 \%, P=0.005\right.$; Figure $\left.\mathrm{S} 1\right)$.

The TSA revealed that the cumulative Z-curve not only crossed the conventional boundary value but also crossed the TSA monitoring boundary. Furthermore, the RIS size of 370 was reached for the incidence of agitation (Figure 5); and the TSA-adjusted $95 \%$ CIs was $0.35-0.68$. TSA of the pooled meta-analysis showed firm evidence that peri-operative administration of dexmedetomidine could significantly reduce the occurrence of EA.

The results of secondary outcomes are listed in Table $\underline{\mathrm{S} 2}$ and sensitivity analysis is shown in Table S3.

\section{Time to emergence, extubation and discharge from PACU}

A total of 7 RCTs (510 participants) recorded the emergence time. ${ }^{17,36,38-42}$ No meta-analysis was performed because of its large clinical heterogeneity $\left(\mathrm{I}^{2}>75 \%\right)$. Extubation was examined in 5 RCTs (380 participants). ${ }^{36,38,40-42}$ There was a set of data that was re-calculated according to source data, ${ }^{40}$ where median and range were transformed to the mean and variance. ${ }^{44}$ However, this group of data was the main source of heterogeneity according to sensitivity analysis, which is why it was excluded from further analysis. The pooled effect demonstrated that dexmedetomidine compared to placebo might prolong extubation time (SMD 0.26, $P=0.03, \mathrm{I}^{2}=0 \%$ ). As for the length of PACU stay, it was reported in seven RCTs (473 patients). ${ }^{2,36-40}$ Conventional meta-analysis suggested that dexmedetomidine did not significantly increase the length of PACU stay (SMD $-0.12, P=0.35, \mathrm{I}^{2}=43 \%$ ).

\section{Analgesics requirement in PACU}

Seven trial studies reported on the number of patients requiring rescue analgesia. ${ }^{17,36-38,40,42,43}$ Dexmedetomidine 


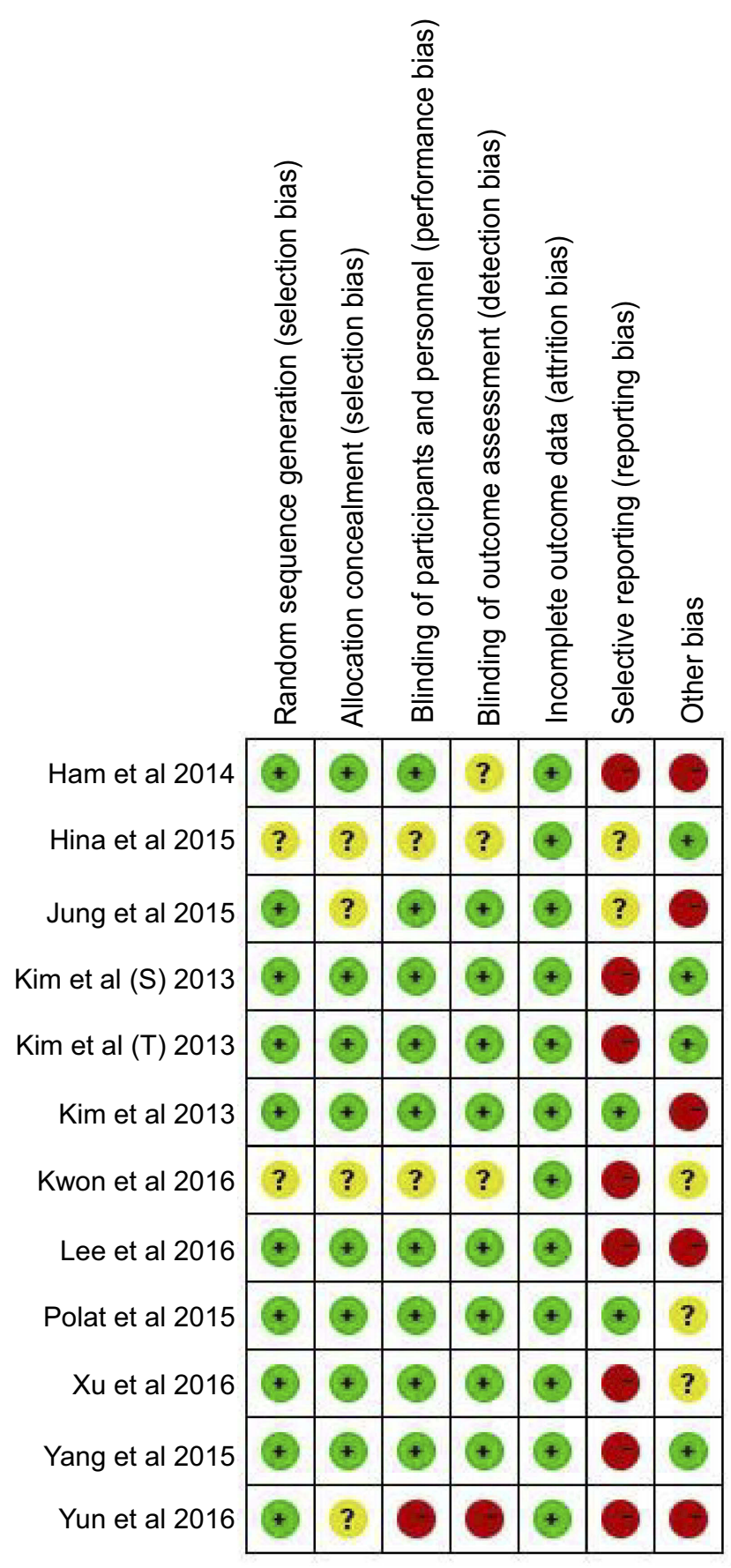

Figure 2 Risk of bias in the included randomized controlled trials.

might reduce the degree of postoperative pain and the number of patients requiring analgesics in PACU (RR $0.66, P=0.0009, \mathrm{I}^{2}=0 \%$; Table S4).

\section{Adverse effects}

Adverse effects included residual sedation,,${ }^{17,36,38,43}$ hypotension, ${ }^{17,18,36,38-43}$ bradycardia ${ }^{18,36,38-40,42,43}$ and postoperative nausea and vomiting (PONV)., ${ }^{2,17,18,36-43}$ The pooled results demonstrated that dexmedetomidine could increase the incidence of hypotension $(\mathrm{RR}=2.37$, $P=0.03, \mathrm{I}^{2}=34 \%$ ). Besides, dexmedetomidine did not have a significant effect on residual sedation $(P=0.24)$, even though the Ramsay sedation score was increased in patients. Moreover, there was no significant reduction in the incidence of PONV in the dexmedetomidine group $(P=0.10)$, as well as the incidence of bradycardia $(P=0.05)$.

\section{Discussion}

Conventional meta-analysis of random-effects models showed that dexmedetomidine could decrease the incidence of EA after general anesthesia in adults (RR 0.49, TSA-adjusted $95 \%$ CI $0.35-0.68, P<0.00001)$. Sensitivity analysis suggested that the results of the meta-analysis were robust. In addition, TAS showed that the information size of the study reached RIS (370). According to the current data, the peri-operative intravenous dexmedetomidine might have a significant effect in the prevention of EA compared with placebo in adults.

There remains lots of queries and incomprehension about EA. So far, the etiology of EA deriving from numerous factors has been described, including male gender, inhalational anesthetics, pain, otolaryngological surgery and the presence of a tracheal tube and/or urinary catheter. ${ }^{6}$ And we found several of these factors such as all male patients included, inhalational anesthetics administration and otolaryngological surgery existed in the trial of Jung et al, resulting in a high incidence of EA (94.7\%) in control group. ${ }^{37}$ Thus, various drugs and techniques have been applied to control EA. Arthura D. Moore et al found that adjunctive agents can be rated in the following order of most effective to least effective interventions: dexmedetomidine, fentanyl, ketamine, clonidine and propofol bolus at the end of sevoflurane-based anesthesia. ${ }^{45}$

Dexmedetomidine exhibits sedative, anxiolytic and analgesic properties by inhibiting the release of norepinephrine mediated through alpha-2 adrenergic receptors in the locus ceruleus and spinal cord. ${ }^{46}$ Moreover, it could not lead to respiratory depression or excessive sedation through accumulation, as propofol and midazolam did. Therefore, dexmedetomidine was used not only as an ideal short-term sedative for patients with mechanical ventilation in intensive care units but also for the prevention or treatment of postoperative agitation and delirium. ${ }^{47-49}$

The current meta-analysis showed that the number of patients requiring rescue analgesia in dexmedetomidine group was significantly decreased, just as VAS scores. Nevertheless, pain itself resulted as a predisposing factor 

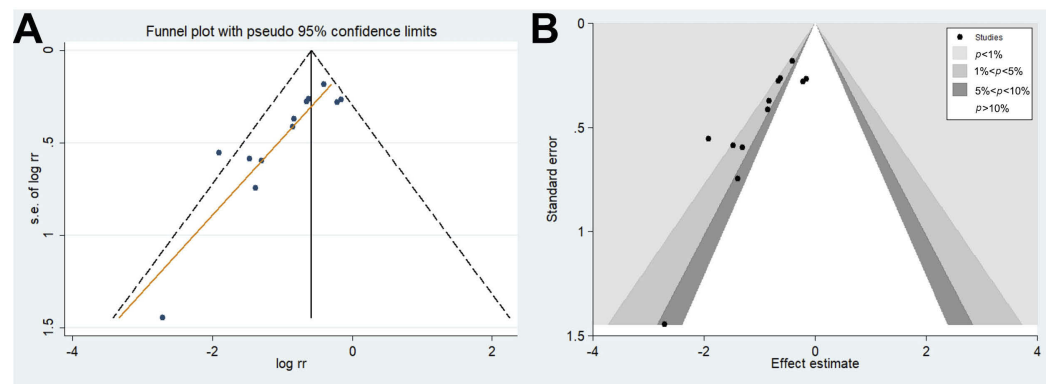

Figure 3 (A) Funnel plot from all trials. The horizontal axis expresses the logarithm of the risk ratio (logrr), and the vertical axis expresses the inverted SE. Asymmetry and a positive association between inverted SE and observed effect sizes are evident. A regression line with SE regressed on effect size is embedded. (B) Contour-enhanced funnel plot. The horizontal axis expresses the log rr, and the vertical axis expresses the inverted SE. Regions of statistical significance are indicated with different colors with white suggesting areas of negative findings. There are less small nonpositive studies (studies in the white area), indicating publication bias may be the reason for the smallstudy effects.

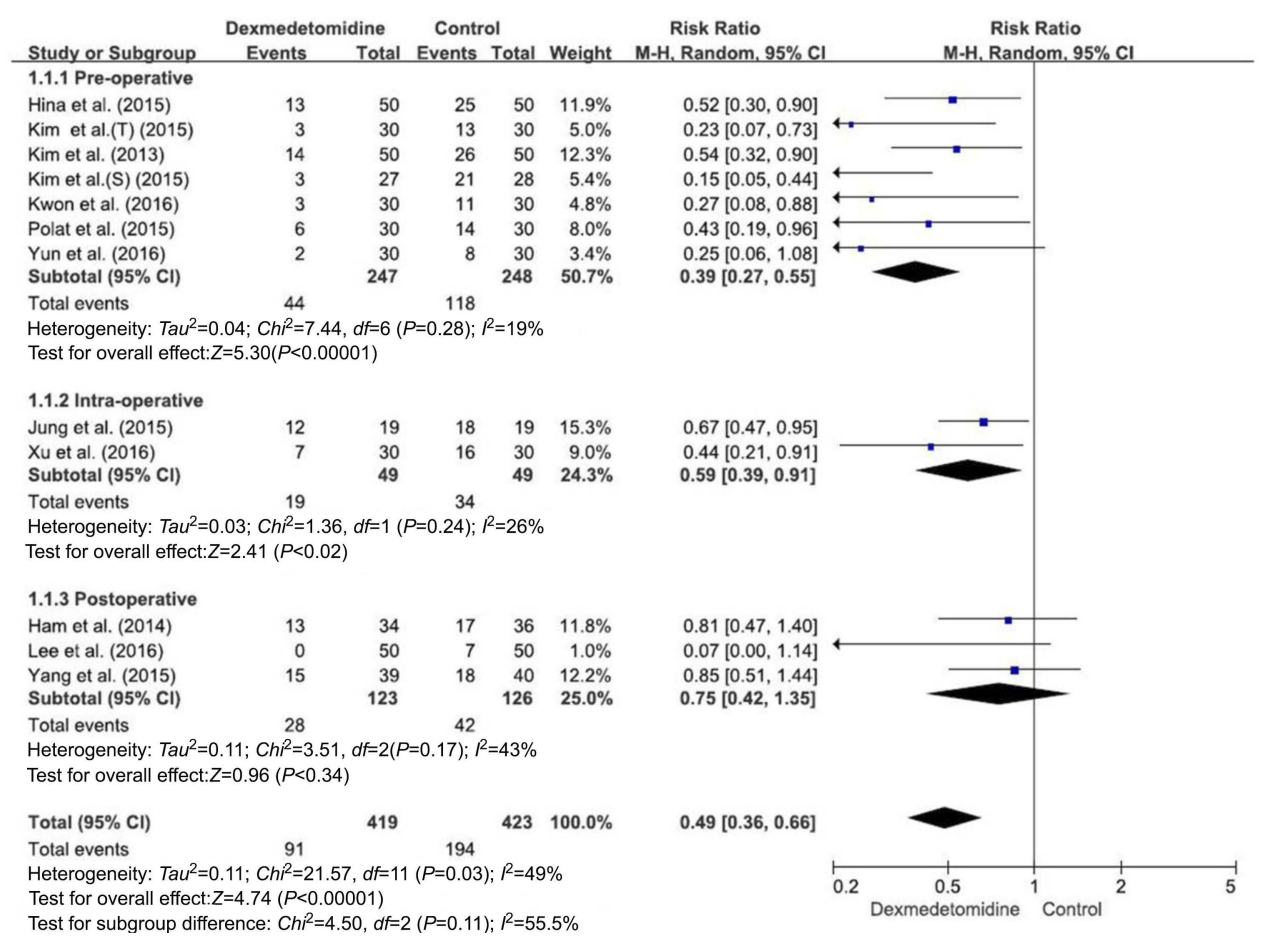

Figure 4 Subgroup analysis of timing of administration of dexmedetomidine.

for the development of EA, ${ }^{6,50}$ thus suggesting that the agitation-sparing pharmacoprophylaxis could provide a new strategy to reduce postoperative pain, which would be a better alternative to increasing putative agitationenhancing analgesic drugs.

In addition, dexmedetomidine exerts hypnotic properties by activating the endogenous sleep-promoting pathway. ${ }^{51}$ A series of studies has indicated that dexmedetomidine significantly improves the subjective sleep quality for ICU patients after surgery. ${ }^{49,52}$ We speculated that dexmedetomidine produced a state akin to natural sleep that stabilized the recovery time of the central nervous system, thereby reducing the occurrence of agitation, which is more optimal approach compared to the use of sedative-hypnotics (such as benzodiazepines) that may increase the incidence of emergence delirium. ${ }^{53}$

Conventional meta-analysis demonstrated that extubation time in the dexmedetomidine group was delayed, while the sensitivity analysis showed that these results were not robust. A latest published study showed that intravenous dexmedetomidine did not affect postoperative extubation time. ${ }^{54}$ Since there were not enough data 


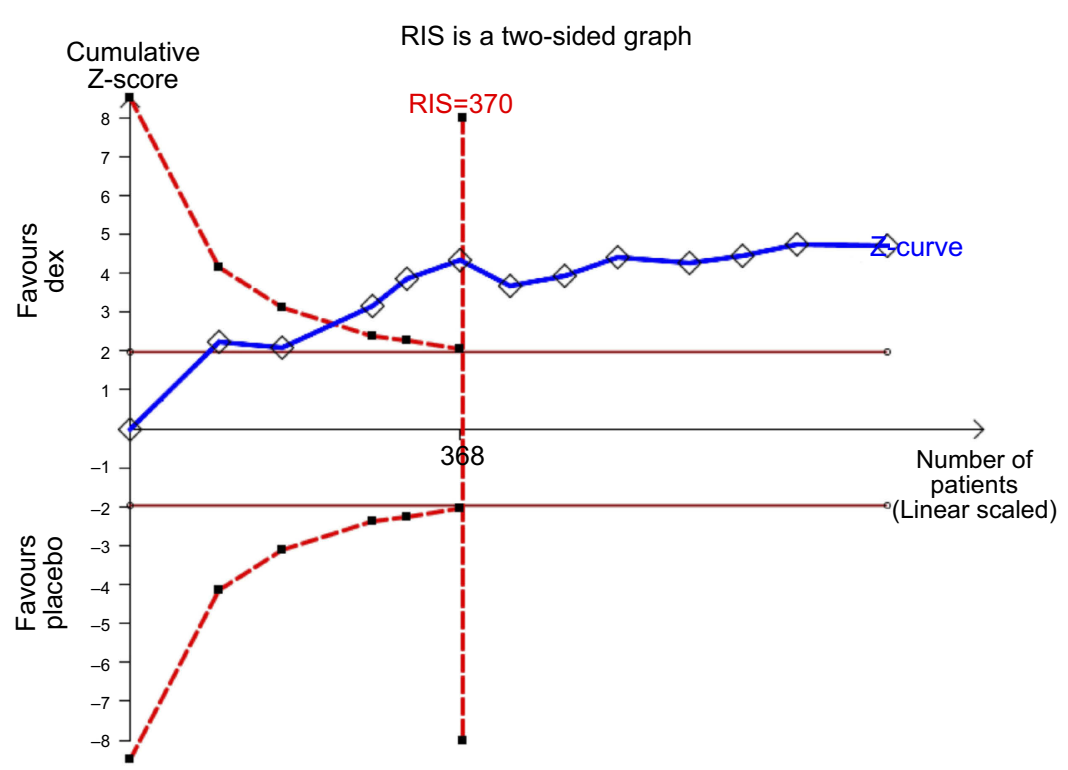

Figure 5 Trial sequential analysis of the effect of dexmedetomidine on the prevention of emergence agitation in adults compared with placebo. We used two-sided tests with type I error set at $5 \%$ and power set at $90 \%$. The variance was calculated from the data obtained from the included trials. Dexmedetomidine significantly reduced the incidence of emergence agitation. The blue line is the cumulative $Z$ curve, and each square frame on the dot represents $I$ trial. The red horizontal line represents a $Z$ score of +1.96 and -1.96 , indicating a conventional significant $P$-value of 0.05 . The red inward lines are the trial sequential monitoring boundaries. A total of 842 patients were analyzed, and the blue line crossed the monitoring boundary before the number reached the required information size (TSA=370), which suggested that there was a significant difference and no more clinical trials were required to prove the hypothesis.

available and substantial clinical heterogeneity was observed, the effect of dexmedetomidine on extubation time remained to be confirmed by the future studies.

The results of adverse effects in our analysis should be cautiously interpreted. What attracted attention was that bradycardia we analyzed was increased by almost 5 times in dexmedetomidine group. Conventional meta-analysis demonstrated that dexmedetomidine did not significantly increase the incidence of residual sedation, bradycardia, PONV, as well as hypotension. Nevertheless, there were not enough details to clarify what each author interpreted as unfavorable events, which had a major influence on the results of our study. Apart from this, data on adverse events were sparse in all studies, while some studies mentioned that no adverse effects were collected, which was also known as "zero event". Considering all of this, it was not possible to make any conclusions regarding side effects of dexmedetomidine because of the lack of information. More clinical trials and samples are needed to verify the safety of dexmedetomidine.

Since elderly people have a higher risk of postoperative cognitive dysfunction, they have also attracted greater attention among researchers. ${ }^{55}$ Latest guidelines on postoperative delirium have clarified that for emergence immediately after surgery, agitation scales such as the RASS were supposed to be used, which meant the early diagnosis and treatment for cognitive impairment at any time after surgery. ${ }^{15}$ Accordingly, we extracted the age range according to the inclusion criteria and data reported in result section for our subgroup analysis. Regrettably, subgroup analysis showed that there was no significant difference between the subgroups. Kim et al reported that EA occurred more frequently among young people which might be related to the elderly having slower metabolism of sedative drugs and having a higher pain threshold. ${ }^{56}$ The current subgroup analysis showed that compared with placebo, dexmedetomidine might have a significant effect in the prevention of EA whatever in the elderly or in the non-elderly.

Nowadays, anesthesiologists focus on the route of administration of dexmedetomidine expecting to find a more optimal administration mode. Our results showed that the timing of administration of dexmedetomidine was the main source of heterogeneity among studies. After conducting regression analysis and subgroup analysis, we found that intravenous dexmedetomidine at the end of surgery did not benefit restlessness during the recovery period, while a recent prospective multicenter RCT revealed that postoperative dexmedetomidine could significantly reduce the occurrence of EA. ${ }^{57}$ We found that Ham et al have added low-dose remifentanil to the experimental group and control group. ${ }^{17}$ 
Moreover, Lee and colleagues have used opioids PCA after surgery, ${ }^{40}$ which contributed to a lower incidence of EA. ${ }^{58}$

\section{Limitations}

We were expecting to find that dexmedetomidine used with different doses and at different time could bring about different effects, but subgroup analysis was not conducted due to the small number of documents and different clinical designs. All the included RCTs were small sample and single center studies, which might have led to the risk of overestimating the estimated effect and underestimating the adverse effects. The misalignment of the funnel plot potentially occurred due to small sample tests and true heterogeneity $\left(\mathrm{I}^{2}=49 \%\right)$, rather than publication bias. In addition, quite a few included trials had uncertain allocation concealment, which was the bias domain most consistently suggested as the source of bias effect. ${ }^{59}$ We did not perform a detailed search for the secondary outcomes. Therefore, there was a possibility of insufficient analysis.

\section{Conclusion}

The meta-analysis showed that peri-operative intravenous dexmedetomidine significantly reduced the incidence of EA and decreased the number of patients in need of rescue analgesia. As for other recovery outcomes such as emergence time, extubation time, length of PACU stay and adverse reactions, they were not useful for drawing any final conclusions and more researches are required to justify it.

\section{Acknowledgments}

The authors thank Jun Cheng (Kidney Disease Center, the First Affiliated Hospital, College of Medicine, Zhejiang University) and Duo Lv (Research Center of Clinical Pharmacy, State Key Laboratory for Diagnosis and Treatment of Infectious Disease, the First Affiliated Hospital, Zhejiang University) for their assistance during the literature search, data collection and analysis. This work was financially supported by grants from National Natural Science Foundation of China (81471171 \& 81771194).

\section{Disclosure}

The authors declare no conflicts of interest in this work.

\section{References}

1. Pickard A, Davies P, Birnie K, Beringer R. Systematic review and meta-analysis of the effect of intraoperative alpha(2)-adrenergic agonists on postoperative behaviour in children. Br J Anaesth. 2014;112 (6):982-990. doi:10.1093/bja/aeu093

2. Kim DJ, Kim SH, So KY, Jung KT. Effects of dexmedetomidine on smooth emergence from anaesthesia in elderly patients undergoing orthopaedic surgery. BMC Anesthesiol. 2015;15:139. doi:10.1186/ s12871-015-0044-6

3. Eckenhoff JE, Kneale DH, Dripps RD. The incidence and etiology of postanesthetic excitment. A clinical survey. Anesthesiology. 1961;22:667-673. doi:10.1097/00000542-196109000-00002

4. Munk L, Andersen G, Moller AM. Post-anaesthetic emergence delirium in adults: incidence, predictors and consequences. Acta Anaesthesiol Scand. 2016;60(8):1059-1066. doi:10.1111/aas.12717

5. Lepouse C, Lautner CA, Liu L, Gomis P, Leon A. Emergence delirium in adults in the post-anaesthesia care unit. $\mathrm{Br} J$ Anaesth. 2006;96(6):747-753. doi:10.1093/bja/ael094

6. Yu D, Chai W, Sun X, Yao L. Emergence agitation in adults: risk factors in 2,000 patients. Can J Anaesth. 2010;57(9):843-848. doi:10.1007/s12630-010-9338-9

7. Sharma PT, Sieber FE, Zakriya KJ, et al. Recovery room delirium predicts postoperative delirium after hip-fracture repair. Anesth Analg. 2005;101(4):1215-1220. doi:10.1213/01.ane.0000167383.44 984.e5

8. Radtke FM, Franck M, MacGuill M, et al. Duration of fluid fasting and choice of analgesic are modifiable factors for early postoperative delirium. Eur J Anaesthesiol. 2010;27(5):411-416. doi:10.1097/ EJA.0b013e3283335cee

9. Kanaya A. Emergence agitation in children: risk factors, prevention, and treatment. J Anesth. 2016;30(2):261-267. doi:10.1007/s00540015-2098-5

10. Kwak KH. Emergence agitation/delirium: we still don't know. Korean J Anesthesiol. 2010;59(2):73-74. doi:10.4097/kjae.2010.59. 2.73

11. Gerlach AT, Murphy CV, Dasta JF. An updated focused review of dexmedetomidine in adults. Ann Pharmacother. 2009;43(12):20642074. doi:10.1345/aph.1M310

12. Afonso J, Reis F. Dexmedetomidine: current role in anesthesia and intensive care. Braz J Anesthesiol. 2012;62(1):118-133. doi:10.1016/ S0034-7094(12)70110-1

13. Fang X-Z, Gao J, Ge Y-L, Zhou L-J, Zhang Y. Network metaanalysis on the efficacy of dexmedetomidine, midazolam, ketamine, propofol, and fentanyl for the prevention of sevoflurane-related emergence agitation in children. Am J Ther. 2016;23(4):e1032-e1042. E1032-E1042. doi:10.1097/MJT.0000000000000321

14. Liu X, Xie G, Zhang K, et al. Dexmedetomidine vs propofol sedation reduces delirium in patients after cardiac surgery: a meta-analysis with trial sequential analysis of randomized controlled trials. J Crit Care. 2016;38:190-196. doi:10.1016/j.jcrc.2016.10.026

15. Aldecoa C, Bettelli G, Bilotta F, et al. European Society of Anaesthesiology evidence-based and consensus-based guideline on postoperative delirium. Eur J Anaesthesiol. 2017;34(4):192-214. doi:10.1097/EJA.0000000000000594

16. Lee HS, Yoon HY, Jin HJ, Hwang SH. Can dexmedetomidine influence recovery profiles from general anesthesia in nasal surgery? Otolaryngol Head Neck Surg. 2018;158(1):43-53. doi:10.1177/ 0194599817733735

17. Ham SY, Kim JE, Park C, Shin MJ, Shim YH. Dexmedetomidine does not reduce emergence agitation in adults following orthognathic surgery. Acta Anaesthesiol Scand. 2014;58(8):955-960. doi:10.1111/aas.12379

18. Yang X, Li Z, Gao C, Liu R. Effect of dexmedetomidine on preventing agitation and delirium after microvascular free flap surgery: a randomized, double-blind, control study. J Oral Maxillofac Surg. 2015;73(6):1065-1072. doi:10.1016/j.joms.2015.01.011 
19. Liberati A, Altman DG, Tetzlaff J, et al. The PRISMA statement for reporting systematic reviews and meta-analyses of studies that evaluate healthcare interventions: explanation and elaboration. $\mathrm{Br} \mathrm{Med} \mathrm{J}$ (Clin Res Ed). 2009;339:b2700. doi:10.1136/bmj.b2651

20. Moller AM, Myles PS. What makes a good systematic review and metaanalysis? Br J Anaesth. 2016;117(4):428-430. doi:10.1093/bja/aew264

21. Higgins, JPT GS Cochrane handbook for systematic reviews of interventions version 5.1.0. cochrane collaboration, 2011. Available from: http://handbookcochraneorg. Accessed July 25, 2016.

22. Higgins JP, Altman DG, Gotzsche PC, et al. The cochrane collaboration's tool for assessing risk of bias in randomised trials. $\mathrm{Br}$ Med $J$ (Clin Res Ed). 2011;343:d5928. doi:10.1136/bmj.d5928

23. DerSimonian R, Kacker R. Random-effects model for meta-analysis of clinical trials: an update. Contemp Clin Trials. 2007;28(2):105114. doi:10.1016/j.cct.2006.04.004

24. Higgins JP, Thompson SG. Quantifying heterogeneity in a metaanalysis. Stat Med. 2002;21(11):1539-1558. doi:10.1002/sim.1186

25. Higgins JP, Thompson SG, Deeks JJ, Altman DG. Measuring inconsistency in meta-analyses. $\mathrm{Br}$ Med J (Clin Res Ed). 2003;327 (7414):557-560. doi:10.1136/bmj.327.7414.557

26. Brok J, Thorlund K, Gluud C, Wetterslev J. Trial sequential analysis reveals insufficient information size and potentially false positive results in many meta-analyses. J Clin Epidemiol. 2008;61(8):763769. doi:10.1016/j.jclinepi.2007.10.007

27. Thorlund K, Engstrøm J, Wetterslev J, et al. User Manual for Trial Sequential Analysis (TSA). Copenhagen: Denmark Copenhagen Trial Unit, Cent Clin Interv Res;2011. Availbale from: wwwctudk/tsa. Accessed January 12, 2015.

28. van der Tweel I, Bollen C. Sequential meta-analysis: an efficient decision-making tool. Clin Trials. 2010;7(2):136-146. doi:10.1177/ 1740774509360994

29. Wetterslev J, Thorlund K, Brok J, Gluud C. Trial sequential analysis may establish when firm evidence is reached in cumulative metaanalysis. J Clin Epidemiol. 2008;61(1):64-75. doi:10.1016/j. jclinepi.2007.03.013

30. Wetterslev J, Thorlund K, Brok J, Gluud C. Estimating required information size by quantifying diversity in random-effects model meta-analyses. BMC Med Res Methodol. 2009;9:86. doi:10.1186/ 1471-2288-9-86

31. Sterne JAC. Meta-Analysis in Stata: An Updated Collection from the Stata Journal. Texas: Stata Press; 2008:242-254.

32. Egger M, Davey Smith G, Schneider M, Minder C. Bias in metaanalysis detected by a simple, graphical test. $\mathrm{Br}$ Med J (Clin Res Ed). 1997;315(7109):629-634. doi:10.1136/bmj.315.7109.629

33. Peters JL, Sutton AJ, Jones DR, Abrams KR, Rushton L. Contourenhanced meta-analysis funnel plots help distinguish publication bias from other causes of asymmetry. J Clin Epidemiol. 2008;61(10):991996. doi:10.1016/j.jclinepi.2007.11.010

34. Harbord RM, Higgins JPT. Meta-regression in Stata. Stata J. 2008;8 (4):493-519. doi:10.1177/1536867X0800800403

35. Dechartres A, Altman DG, Trinquart L, Boutron I, Ravaud P. Association between analytic strategy and estimates of treatment outcomes in meta-analyses. Jama. 2014;312(6):623-630. doi:10. 1001/jama.2014.8166

36. Kim SY, Kim JM, Lee JH, Song BM, Koo BN. Efficacy of intraoperative dexmedetomidine infusion on emergence agitation and quality of recovery after nasal surgery. Br J Anaesth. 2013;111(2):222228. doi:10.1093/bja/aet056

37. Jung JW, Kim YH, Lee KH, et al. Effect of dexmedetomidine on emergence agitation in male patients undergoing closed reduction of a nasal bone fracture. RMJ. 2015;40(2):191-196.

38. Polat R, Peker K, Baran I, Bumin Aydin G, Topcu Guloksuz C, Donmez A. Comparison between dexmedetomidine and remifentanil infusion in emergence agitation during recovery after nasal surgery: a randomized double-blind trial. BMC Anesthesiol. 2015;64(10):740 746.
39. Kwon SY, Joo JD. Effects of dexmedetomidine infusion on the recovery profiles of patients undergoing transurethral resection. J Korean Med Sci. 2016;31(1):125-130. doi:10.3346/jkms.2016.31.1.125

40. Lee SH, Lee CY, Lee JG, Kim N, Lee HM, Oh YJ. Intraoperative dexmedetomidine improves the quality of recovery and postoperative pulmonary function in patients undergoing video-assisted thoracoscopic surgery: a CONSORT-prospective, randomized, controlled trial. Medicine. 2016;95(7):e2854. doi:10.1097/MD.0000000000004864

41. Li Y, Wang B, Zhang LL, et al. Dexmedetomidine combined with general anesthesia provides similar intraoperative stress response reduction when compared with a combined general and epidural anesthetic technique. Anesth Analg. 2016;122(4):1202-1210. doi:10.1213/ANE.0000000000001165

42. Xu K, Pan Y, Zhu M. Effects of dexmedetomidine on the recovery profiles from general anesthesia in patients undergoing endoscopic sinus surgery. Int J Clin Exp Med. 2016;9(5):8405-8410.

43. Khurshid DH, Muneer DK, Sadiq Malla M. Effect of dexmedetomidine on emergence agitation after nasal surgeries. Med Sci Clin Res. 2015;3(9):7527-7531.

44. Hozo SP, Djulbegovic B, Hozo I. Estimating the mean and variance from the median, range, and the size of a sample. BMC Med Res Methodol. 2005;5:13. doi:10.1186/1471-2288-5-27

45. Moore AD, Anghelescu DL. Emergence delirium in pediatric anesthesia. Paediatr Drugs. 2017;19(1):11-20. doi:10.1007/s40272016-0201-5

46. Maze M, Scarfini C, Cavaliere F. New agents for sedation in the intensive care unit. Crit Care Clin. 2001;17(4):881-897.

47. Djaiani G, Silverton N, Fedorko L, et al. Dexmedetomidine versus propofol sedation reduces delirium after cardiac surgery: a randomized controlled trial. Anesthesiology. 2016;124(2):362-368. doi:10.1097/ALN.0000000000000951

48. Reade MC, Eastwood GM, Bellomo R, et al. Effect of dexmedetomidine added to standard care on ventilator-free time in patients with agitated delirium: a randomized clinical trial. Jama. 2016;315 (14):1460-1468. doi:10.1001/jama.2016.2707

49. Su X, Meng ZT, Wu XH, et al. Dexmedetomidine for prevention of delirium in elderly patients after non-cardiac surgery: a randomised, double-blind, placebo-controlled trial. Lancet. 2016;388 (10054):1893-1902. doi:10.1016/S0140-6736(16)30580-3

50. Kosar CM, Tabloski PA, Travison TG, et al. Effect of preoperative pain and depressive symptoms on the development of postoperative delirium. Lancet Psychiatry. 2014;1(6):431-436. doi:10.1016/S22150366(14)00006-6

51. Nelson LE, Lu J, Guo T, Saper CB, Franks NP, Maze M. The alpha2-adrenoceptor agonist dexmedetomidine converges on an endogenous sleep-promoting pathway to exert its sedative effects. Anesthesiology. 2003;98(2):428-436. doi:10.1097/00000542200302000-00024

52. Wu XH, Cui F, Zhang C, et al. Low-dose dexmedetomidine improves sleep quality pattern in elderly patients after noncardiac surgery in the intensive care unit: a pilot randomized controlled trial. Anesthesiology. 2016;125(5):979-991. doi:10.1097/ALN.000000000 0001325

53. Coull JT, Jones ME, Egan TD, Frith CD, Maze M. Attentional effects of noradrenaline vary with arousal level: selective activation of thalamic pulvinar in humans. NeuroImage. 2004;22(1):315-322. doi:10.1016/j.neuroimage.2003.12.022

54. Jo YY, Kim HS, Lee KC, Chang YJ, Shin Y, Kwak HJ. Consort the effect of intraoperative dexmedetomidine on hemodynamic responses during emergence from nasotracheal intubation after oral surgery. Medicine. 2017;96(16):e6661. doi:10.1097/MD.000 0000000006661

55. OB H, Mohan H, Hare CO, Reynolds JV, Kenny RA. Mind over matter? The hidden epidemic of cognitive dysfunction in the older surgical patient. Ann Surg. 2017;265(4):677-691. doi:10.1097/ SLA.0000000000001900 
56. Kim HJ, Kim DK, Kim HY, Kim JK, Choi SW. Risk factors of emergence agitation in adults undergoing general anesthesia for nasal surgery. Clin Exp Otorhinolaryngol. 2015;8(1):46-51. doi:10.3342/ceo.2015.8.1.46

57. Aouad MT, Zeeni C, Al Nawwar R, et al. Dexmedetomidine for improved quality of emergence from general anesthesia: a dose-finding study. Anesth Analg. 2017. doi:10.1213/ANE.0000000000002763
58. Tan Y, Shi Y, Ding H, Kong X, Zhou H, Tian J. Mu-Opioid agonists for preventing emergence agitation under sevoflurane anesthesia in children: a meta-analysis of randomized controlled trials. Paediatr Anaesth. 2016;26(2):139-150. doi:10.1111/pan.12815

59. Sedgwick P. Meta-analysis: testing for reporting bias. $\mathrm{Br}$ Med J (Clin Res Ed). 2015;350:g7857.

\section{Publish your work in this journal}

Drug Design, Development and Therapy is an international, peerreviewed open-access journal that spans the spectrum of drug design and development through to clinical applications. Clinical outcomes, patient safety, and programs for the development and effective, safe, and sustained use of medicines are a feature of the journal, which has also

been accepted for indexing on PubMed Central. The manuscript management system is completely online and includes a very quick and fair peer-review system, which is all easy to use. Visit http://www. dovepress.com/testimonials.php to read real quotes from published authors. 